\title{
RADO'S THEOREM FOR THE LOEB SPACE OF AN INTERNAL *-FINITELY ADDITIVE MEASURE SPACE
}

\author{
BOŠKO ŽIVALJEVIĆ
}

(Communicated by Andreas R. Blass)

\begin{abstract}
A version of Rado's theorem about the existence of a family of subsets of a given family of sets combinatorially similar to another family of sets is proved for the Loeb space of an internal *-finitely additive measure space. As a corollary we obtain the Loeb measured case of the result of B. Bollobas and $\mathrm{N}$. Th. Varopoulos about the existence of a family of mutually disjoint measurable subsets of the given family of measurable sets, having the prescribed measure.
\end{abstract}

The aim of this paper is to prove a version of R. Rado's theorem for the Loeb space of an internal *-finitely additive measure space.

In 1937 R. Rado proved (see [Ra]) an extension of P. Hall's theorem (see [Ha]) about the existence of distinct representatives of a finite family of finite sets. He replaced "number of elements of a set" by a general measure function and instead of selecting single elements from each member of a family he selected subsets combinatorially similar to a given family of sets (see the definition below). Although Rado's theorem deals with a very general measure function taking values in a general linearly ordered set, it seems to be restricted by the assumption of finiteness of the family of sets in question. Here, using the finite form of Rado's theorem, we extend Rado's theorem to an infinite number of measurable sets for the class of Loeb spaces of internal $*$-finitely additive measure spaces.

We assume that the reader is familiar with basic facts of nonstandard analysis. For the necessary definitions and elementary properties of a nonstandard universe refer to $[\mathrm{StBa}]$ or [HuLo].

Let $\mathscr{M}=(\Omega, \Sigma, \mu)$ be an internal, nonatomic, $*$-finitely additive measure space, that is, $\Omega$ is an internal set, $\Sigma$ a $*$-algebra of internal subsets of $\Omega$, and $\mu$ a $*$-finitely additive measure on $\Sigma$. Nonatomic means that for every element $A \in \Sigma$ with $\mu(A)$ not being infinitesimal, there exists a set $B \in \Sigma$ with $B \subseteq A$

Received by the editors May 26, 1989 and, in revised form, May 31, 1990.

1980 Mathematics Subject Classification (1985 Revision). Primary 03H05, 28A99; Secondary 05 C99.

The research was supported by the National Science Foundation and the Research Board of the University of Illinois. 
and $\mu(B)$ not infinitely close to $\mu(A)$. For example, $\Omega$ can be a hyperfinite set, $\Sigma$ the $*$-algebra of all internal subsets of $\Omega$, and $\mu$ a counting measure defined as $\mu(A)=|A / H|$, where $H$ is some hyperfinite integer. To $\mathscr{M}$ (see [Lo]) we can associate a standard, complete measure space $L(\mathscr{M})=(\Omega, L(\Sigma), L(\mu))$, called the Loeb space of $\mathscr{M}$, where $L(\Sigma)$ is a standard $\sigma$-algebra of subsets of $\Omega$ and $L(\mu)$ is a standard $\sigma$-additive measure on $L(\Sigma)$ extending the standard measure $s t \circ \mu$, st being the standard part map. The elements of $L(\Sigma)$ are called Loeb measurable sets.

A positive boolean polynomial $\beta\left(x_{1}, \ldots, x_{n}\right)$ is a term on the language $\{\cap, \cup\}$. In other words, we define every letter $x_{i}(i=1,2, \ldots)$ to be a positive boolean polynomial, and if $\alpha$ and $\beta$ are positive boolean polynomials then $(\alpha \cap \beta)$ and $(\alpha \cup \beta)$ are also positive boolean polynomials. Also, an expression is a positive boolean polynomial if and only if it is obtained by the above procedure. Here, as usual, " $\cup$ " and " $\cap$ " are symbols for union and intersection respectively.

Given two families $\left(M_{i}\right)_{i \in \Lambda}$ and $\left(N_{i}\right)_{i \in \Lambda}$ of Loeb measurable sets, we say that they are combinatorially similar if

$$
L(\mu)\left(\alpha\left(M_{i}\right)\right)=L(\mu)\left(\alpha\left(N_{i}\right)\right)
$$

for every positive boolean polynomial $\alpha$. Here, $\alpha\left(M_{i}\right)$ and $\alpha\left(N_{i}\right)$ are the results of substituting the letter $x_{i}$ in the positive boolean polynomial $\alpha$ by the sets $M_{i}$ and $N_{i}$ respectively. The same definition applies if the measure $L(\mu)$ is replaced by an abstract set function and, in particular, by a countably additive measure. In the last case we formulate an instance of Rado's aforementioned theorem.

Theorem [Ra]. Let $\mathscr{S}=\left(S_{i}\right)_{i=1}^{n}$ and $\mathscr{T}=\left(T_{i}\right)_{i=1}^{n}$ be two finite families of measurable sets of finite measure in a nonatomic measure space $(X, \mathscr{M}, m)$. There exists a family $\mathscr{U}=\left(U_{i}\right)_{i=1}^{n}$ of measurable sets combinatorially similar to the family $\mathscr{T}$ such that $U_{i} \subseteq S_{i}$ for every $i=1, \ldots, n$ if and only if

$$
m\left(\alpha\left(S_{i}\right)\right) \geq m\left(\alpha\left(T_{i}\right)\right)
$$

for every positive boolean polynomial $\alpha$.

Now we state and prove our result.

Theorem I. Let $\left(M_{i}\right)_{i \in \Lambda}$ and $\left(N_{i}\right)_{i \in \Lambda}$ be two families of Loeb measurable sets of finite measure, and suppose that the nonstandard universe is $\max \left\{|\Lambda|^{+}, \omega_{0}\right\}$ saturated. There exists a family $\left(H_{i}\right)_{i \in \Lambda}$ of Loeb measurable subsets of $M_{i}$, $H_{i} \subseteq M_{i}(i \in \Lambda)$ combinatorially similar to $\left(N_{i}\right)_{i \in \Lambda}$ if and only if

$$
L(\mu)\left(\alpha\left(M_{i}\right)\right) \geq L(\mu)\left(\alpha\left(N_{i}\right)\right)
$$

for every positive boolean polynomial $\alpha$.

Proof. Let condition (1) be satisfied. If the index set $\Lambda$ is finite then the result follows from Rado's theorem [Ra]. Assume that $\Lambda$ is infinite. 
Suppose first that $M_{i}=A_{i}, N_{i}=B_{i} \quad(i \in \Lambda)$ are internal. Let $e \subseteq \Lambda$ be a finite subset of $\Lambda$. Using Rado's theorem there exists a sequence of Loeb measurable subsets of $A_{i}(i \in e)$ combinatorially similar to $B_{i}(i \in e)$. For each Loeb measurable set $M$ of finite measure there exists an internal set $A$ with $L(\mu)(M \triangle A)=0$. Also, if $L_{i}(i \in \Lambda)$ is a family of Loeb measurable sets and $E_{i}(i \in \Lambda)$ is a family of internal sets with $L(\mu)\left(L_{i} \Delta E_{i}\right)=0$, and $\alpha$ a positive boolean polynomial then

$$
L(\mu)\left(\alpha\left(L_{i}\right)\right)=L(\mu)\left(\alpha\left(E_{i}\right)\right) .
$$

So, for a given finite $e \subseteq \Lambda$ there exists a sequence $E_{i} \quad(i \in e)$ of internal subsets of $A_{i}$, such that $\left(E_{i}\right)_{i \in e}$ and $\left(B_{i}\right)_{i \in e}$ are combinatorially similar.

For an arbitrary finite subset $e \subseteq \Lambda$, a natural number $n \in \omega$ and a finite set $F$ of positive boolean polynomials let $\mathscr{F}(e, n, F)$ be the set of all sequences $D_{1}, \ldots, D_{m}$ of $m(=|e|)$ internal sets satisfying

$$
\begin{aligned}
\mu\left(D_{i} \backslash A_{i}\right) & \leq 1 / n \quad \\
\mu\left(\alpha\left(D_{i}\right) \Delta \alpha\left(B_{i}\right)\right) & \leq 1 / n \quad(i \in e) ;
\end{aligned}
$$

Between triples $(e, n, F)$ we can introduce a natural ordering $\preceq$ as

$$
(e, n, F) \preceq(f, m, G)
$$

if and only if $e \subseteq f, n \leq m, F \subseteq G$.

Let us extend a function $\mathscr{F}$ to an internal function. The existence of such an extension is guaranteed by $|\Lambda|^{+}$saturation. Then, again by $|\Lambda|^{+}$saturation, there exists a triple $(h, \nu, A)$ such that $(e, n, F) \preceq(h, \nu, A)$ for every standard triple $(e, n, F)$ and such that (2) is satisfied for $(h, \nu, A)$. Let $\bar{H}_{i}$ $(i \in \Lambda)$ be the elements of $A$ with standard indices. Then,

$$
\begin{aligned}
\mu\left(\bar{H}_{i} \backslash A_{i}\right) \leq 1 / \nu & (i \in \Lambda), \\
\mu\left(\alpha\left(\bar{H}_{i}\right) \Delta \alpha\left(B_{i}\right)\right) \leq 1 / \nu & (i \in \Lambda, \alpha \in P),
\end{aligned}
$$

where $P$ is the set of all positive boolean polynomials. That means that if we define $H_{i}=\bar{H}_{i} \cap A_{i} \quad(i \in \Lambda)$ then the family $\left(H_{i}\right)_{i \in \Lambda}$ will be combinatorially similar to the family $\left(B_{i}\right)_{i \in \Lambda}$.

Finally, if $\left(M_{i}\right)_{i \in \Lambda}$ and $\left(N_{i}\right)_{i \in \Lambda}$ are arbitrary Loeb measurable sets the claim follows from the fact that we can take $\left(A_{i}\right)_{i \in \Lambda}$ and $\left(B_{i}\right)_{i \in \Lambda}$ internal with

$$
L(\mu)\left(\left(M_{i} \Delta A_{i}\right)\right)=L(\mu)\left(\left(N_{i} \Delta B_{i}\right)\right)=0
$$

and apply the first half of the proof.

In $[\mathrm{BoV}]$ the authors proved the following result using a variant of P. Hall's theorem and the Krein-Milman theorem.

Theorem [BoV]. Given any nonatomic measure space $(X, \mathscr{A}, m)$, a family $M_{i}$ $(i \in \Lambda)$ of measurable sets of finite measure in $\mathscr{A}$ and a family $a_{i}(i \in \Lambda)$ of real numbers, there exists a family $N_{i}(i \in I)$ of disjoint measurable sets in $\mathscr{A}$ such that

$$
N_{i} \subseteq M_{i}, \quad m\left(N_{i}\right)=a_{i} \quad(i \in \Lambda)
$$


if and only if

$$
m\left(\bigcup_{i \in e} M_{i}\right) \geq \sum_{i \in e} a_{i}
$$

for every finite subset $e \subseteq \Lambda$.

It is not known to me if a result similar to one we proved in Theorem $I$ is still true for an arbitrary, nonatomic measure space. Also, if such a statement is indeed valid, it is not obvious that the result of Bollobas and Varopoulos is its consequence. However, we shall now prove that, in the case of the Loeb space, the above result is indeed a consequence of our Theorem I. First, we need the following lemma that was noticed before by many authors (see [LaRo]), and whose proof we give here for the sake of completeness. We suppose that our nonstandard universe is $\max \left\{|\Lambda|^{+}, \omega_{0}\right\}$ saturated.

Lemma. Let $A_{i}(i \in \Lambda)$ be an infinite sequence of internal sets from $\Sigma$ of $L(\mu)$ measure zero. Then, $\bigcup_{i \in \Lambda} A_{i}$ is of $L(\mu)$ measure zero.

Proof. Let $\varepsilon>0$ be a given and standard real number. For a finite $e \subseteq \Lambda$ let

$$
F_{e}=\left\{A \in \Sigma: \bigcup_{i \in e} A_{i} \subseteq A, \mu(A)<\varepsilon\right\} .
$$

Then, the family $F_{e}\left(e \in[\Lambda]^{\mathrm{fin}}\right)$, where $[\Lambda]^{\mathrm{fin}}$ is the set of all finite subsets of $\Lambda$, possesses the finite intersection property and is of cardinality $|\Lambda|$. Therefore, by $|\Lambda|^{+}$saturation, there exists

$$
A \in \bigcap_{e \in[\Lambda]^{\text {fn }}} F_{e} .
$$

We have $\bigcup_{i \in \Lambda} A_{i} \subseteq A$ and $\mu(A)<\varepsilon$, so $\bigcup_{i \in \Lambda} A_{i}$ is of $L(\mu)$ measure $\leq \varepsilon$. As $\varepsilon$ is arbitrary $\bigcup_{i \in \Lambda} A_{i}$ is of $L(\mu)$ measure 0 .

Corollary. Suppose that our nonstandard universe is $\max \left\{|\Lambda|^{+}, \omega_{0}\right\}$ saturated and let $M_{i}(i \in \Lambda)$ be a sequence of Loeb measurable sets of finite measure in $L(\Sigma)$. Let $a_{i}(i \in \Lambda)$ be a sequence of positive real numbers. Then, there exists a sequence $N_{i}(i \in \Lambda)$ of disjoint Loeb measurable sets such that $N_{i} \subseteq M_{i}$ and $L(\mu)\left(N_{i}\right)=a_{i} \quad(i \in \Lambda)$, if and only if

$$
L(\mu)\left(\bigcup_{i \in e} M_{i}\right) \geq \sum_{i \in e} a_{i}
$$

for every finite $e \subseteq \Lambda$.

Proof. Let $H_{i} \quad(i \in \Lambda)$ be a sequence of mutually disjoint Loeb measurable sets in $L(\Sigma)$ with $L(\mu)\left(H_{i}\right)=a_{i} \quad(i \in \Lambda)$. Then, condition (3) implies that condition (1) of Theorem I is satisfied for the sequences $\left(M_{i}\right)_{i \in \Lambda}$ and $\left(H_{i}\right)_{i \in \Lambda}$. Therefore, by Theorem I, there exists a sequence $\bar{N}_{i}(i \in \Lambda)$ of Loeb sets combinatorially similar to $\left(H_{i}\right)_{i \in \Lambda}$ and such that $\bar{N}_{i} \subseteq M_{i}(i \in \Lambda)$. That 
means that $L(\mu)\left(\bar{N}_{i} \cap \bar{N}_{j}\right)=0$ for every distinct $i, j \in \Lambda$ and $L(\mu)\left(\bar{N}_{i}\right)=$ $a_{i} \quad(i \in \Lambda)$. Let $A_{i} \quad(i \in \Lambda)$ be a sequence of internal sets from $\Sigma$ with $L(\mu)\left(A_{i} \Delta \bar{N}_{i}\right)=0 \quad(i \in \Lambda)$. Then, $L(\mu)\left(A_{i} \cap A_{j}\right)=0$ for every two distinct $i, j \in \Lambda$. By our lemma, the sets $B_{i}=A_{i} \backslash\left(\bigcup_{j \neq i} A_{j}\right)$ are mutually disjoint and $L(\mu)$ measurable with $L(\mu)\left(B_{i} \Delta \bar{N}_{i}\right)=0 \quad(i \in \Lambda)$. Therefore, if we put $N_{i}=\bar{N}_{i} \cap M_{i} \quad(i \in \Lambda)$ we shall obtain a desired family of sets.

\section{REFERENCES}

[BoV] B. Bollobas and N. Th. Varopoulos, Representation of systems of measurable sets, Math. Proc. Cambridge Philos. Soc. 78 (1974), 323-325.

[Ha] P. Hall, On representatives of subsets, J. London Math. Soc. 10 (1935), 26-30.

[HuLo] A. E. Hurd and P. A. Loeb, An introduction to nonstandard real analysis, Academic Press, New York, 1985.

[LaRo] D. Landers and L. Rogge, Universal Loeb-measurability of sets and of the standard part map with applications, Trans. Amer. Math. Soc. 304 (1983), 229-243.

[Lo] P. A. Loeb, Conversion from nonstandard to standard measure space and applications in probability theory, Trans. Amer. Math. Soc. 211 (1975), 113-122.

[Ra] R. Rado, $A$ theorem on general measure function, Proc. London Math. Soc. 44 (1938), 61-91.

[StBa] K. D. Stroyan and J. M. Bayod, Foundations of infinitesimal stochastic analysis, NorthHolland, Amsterdam, 1986.

Department of Mathematics, University of Illinois, Urbana, Illinois 61801

Current address: Department of Mathematics, University of Sarajevo, V. Putnika 43, 71000 Sarajevo, Yugoslavia 